\title{
ANTIDIABETIC EFFECT OF EXTRACTS OF BLUMEA LACERA DC. IN STREPTOZOTOCIN INDUCED HYPERGLYCEMIC RATS
}

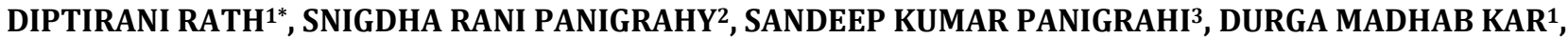 \\ LAXMIDHAR MAHARANA ${ }^{1}$
}

1Department of Pharmacology, School of Pharmaceutical Sciences, Siksha '0' Anusandhan University, Kalinga Nagar, Bhubaneswar 751003, Odisha, India, ${ }^{2}$ Department of Pharmacology, MKCG Medical College and Hospital, Brahmapur 760004, Odisha, India, ${ }^{3}$ Department of Community Medicine, IMS and SUM Hospital, Siksha ‘O’ Anusandhan University, Kalinga Nagar, Bhubaneswar 751003, Odisha, India

Email: diptiranirath@soauniversity.ac.in

Received: 27 Sep 2016 Revised and Accepted: 31 Aug 2017

\section{ABSTRACT}

Objective: Present study of this research was undertaken to evaluate the antidiabetic activities of extracts of aerial parts of Blumea lacera DC. (Asteraceae) in streptozotocin (STZ) induced hyperglycemic rat.

Methods: The methanol extracts (MEBL) and aqueous extract (AEBL) of B. lacera DC. were investigated in streptozotocin (STZ) induced hyperglycemic rats at a dose level of 200 and $400 \mathrm{mg} / \mathrm{kg}$ body weight, in oral glucose tolerance test (OGTT), acute and subacute antidiabetic (30 d) models keeping a parallel group of metformin $(250 \mathrm{mg} / \mathrm{kg}$ body weight) as standard drug. The serum biochemical parameters, histopathology of liver and pancreas were examined and analyzed statistically.

Results: Treatment with methanol extract of B. lacera (MEBL) at a dose of $200 \mathrm{mg} / \mathrm{kg}$ and $400 \mathrm{mg} / \mathrm{kg}$ body weight, significantly decrease (p<0.05) blood glucose level from $289.83 \pm 9.83$ and $289.83 \pm 2.71$ to $201.83 \pm 8.87$ and $105.00 \pm 2.05$ respectively with corresponding percentage fall of blood glucose to $30.40 \pm 1.79$ and $63.78 \pm 0.59$. It also improved the glycated haemoglobin (HbA1c) near to normal value, restored the lipid and bio-chemical level and rejuvenate beta cells of pancreas, thereby improve insulin secretion.

Conclusion: The result of the present study concluded that extracts of B. lacera DC possess marked antidiabetic activity. However, methanol extract of B. lacera (MEBL) at the dose of $400 \mathrm{mg} / \mathrm{kg}$ showed significant dose dependent antidiabetic effects without any destruction and restores the structure of liver and pancreas of hyperglycemic rats.

Keywords: Blumea lacera, Metformin, Blood glucose level, Bio-chemical level, Insulin, Glycated hemoglobin

(C) 2017 The Authors. Published by Innovare Academic Sciences Pvt Ltd. This is an open access article under the CC BY license (http://creativecommons.org/licenses/by/4.0/) DOI: http://dx.doi.org/10.22159/ijpps.2017v9i10.19851

\section{INTRODUCTION}

Diabetes mellitus (DM) is a chronic metabolic disorder associated with disturbance of carbohydrate, protein, fat metabolism. The incidence and prevalence of diabetes in adults is expected to raise from 135 million in 1995 to 300 million in the year 2025 [1]. Apart from synthetic medicines, herbal drugs are also gaining importance day by day due to their effectiveness, low cost and minimal side effects [2, 3].

Blumea lacera (Burm. f.) DC (Asteraceae), also equally known as Conyza lacera, is an annual herb found in all parts of India. It is covered with white and silky hair. It is also known as kakaronda, siyalmutra, susksampatra, bonomula and shealmoti. It is mentioned in Ayurveda as bitter, acrid, thermogenic and astringent with camphoraceous smelling. Ethno botanically, the plant is well known for its anti-inflammatory, diuretic, antioxidant, anthelmintic, antipyretic, antimicrobial, expectorant, ophthalmic, febrifuge and digestive properties in many parts of the country $[4,5]$. The methanol extracts of the leaves of Blumea eriantha DC. Showed anti-hyperglycemic activity in streptozotocin (STZ) induced diabetic rats and also protects against liver and renal damage [6]. The methanol leaf extract of this plant was preliminarily evaluated for its oral glucose tolerance tests in mice model [7]. It was also reported to have $\alpha$-amylase inhibitory activity using starch-iodine method [8]. No report was established regarding the in vivo antidiabetic effects of B. lacera and hence, the present study was undertaken to evaluate the antidiabetic effect of extracts of B. lacera in streptozotocin (STZ) induced hyperglycemic rats.

\section{MATERIALS AND METHODS}

Collection of plant materials and preparation of plant extracts

The aerial parts of B. lacera were collected from two adjacent villages in the sub-urban area of Bhubaneswar, the capital city of
Odisha (India) in the month of January. The authentication of plant was done by a botanist at Regional plant resource center, Bhubaneswar. After collection, they were washed with distilled water and then shed dried for two $\mathrm{w}$ at room temperature. The plant materials were then grinded into coarse powder by a mechanical grinder. A voucher specimen was (SPS/SOAU - 08) stored at herbarium of our institute for future need. Powdered drugs were defatted using petroleum ether in soxhlet apparatus. Methanol and aqueous extracts were then prepared by cold maceration process (for $72 \mathrm{~h}$ ) with occasional shakings, using their respective diluents. It was then filtered using whatman filter paper followed by evaporation sequentially by rotary evaporator and water bath. These were then stored in vacuum desiccator for further use. The percentage yield of methanol and aqueous extracts with respect to dried powder were $15.8 \% \mathrm{w} / \mathrm{w}$ and $19.1 \% \mathrm{w} / \mathrm{w}$ respectively.

\section{Phytochemical screening}

The qualitative phytochemical screening of B. lacera were carried out according to the standard procedure which revealed the presence or absence of carbohydrates (Molisch's test), reducing sugar (Fehling's test), saponins (Frothing test), tannins ( $\mathrm{FeCl}_{3}$ test), flavonoids (Schinoda's test), steroids (Salkowski test), glycosides (Keller Killiani Tests), alkaloid (Wagner's reagent test) and terpenoids (Salkowski test) etc. $[9,10]$.

\section{Experimental animals}

Healthy Swiss albino male rats weighing about $250-300$ g aged about $4-5$ mo were procured from the animal house of the institute and housed in polycarbonate cages. They were given free access to water ad libitum and provided with standard pellet diet. Before conducting any experiment, they were acclimatized to the 
experimental conditions and deprived of food for at least $10-12 \mathrm{~h}$. The room temperature was maintained at $25-30{ }^{\circ} \mathrm{C}$ and relative humidity at $45-55 \%$ as per the standard procedure. The animals having blood glucose level in the normal range i.e. $66-110 \mathrm{mg} / \mathrm{dl}$ were included in this study.

\section{Approval from animal ethical committee}

Approval for animal testing was taken from Institutional Animal Ethical Committee (IAEC). The letter-number from ethical committee was 57/SPS/IAEC/SOAU dated 27 ${ }^{\text {th }}$ December 2013.

\section{Acute toxicity study}

According to the principles of Organisation for economic cooperation and development (OECD guidelines 423), acute oral toxicity test was performed on $12 \mathrm{~h}$ fasted healthy albino rats to rule out any toxicity of the extracts before conducting the whole study. Extracts were administered to both test drugs treated groups (50 $\mathrm{mg} / \mathrm{kg}$ to a maximum of $4000 \mathrm{mg} / \mathrm{kg}$ body weight) while control group received only vehicle (Distilled water+Tween 80 ). Test and control groups of animals were critically observed for change in behaviour and acute signs of toxicity, beginning from $30 \mathrm{~min}$ of extract administration till $4 \mathrm{~h}$. Then the rats were occasionally observed up to $72 \mathrm{~h}$ for any remarkable toxic effects, followed by rare observation till $14 \mathrm{~d}$ for any mortality [11].

\section{Induction of diabetes}

Diabetes was induced by single intraperitoneal injection of streptozotocin $40 \mathrm{mg} / \mathrm{kg}$ body weight mixed with $0.1 \mathrm{M}$ Citrate buffer (PH 4.5), prepared immediately before induction, to the fasted rats. After 12 to $14 \mathrm{~d}$ of streptozotocin (STZ) induction, animals having blood glucose level above $250 \mathrm{mg} / \mathrm{dl}$ were considered stable and included in our experiment as diabetic rats $[9,10]$.

\section{Design of experimental study}

A total of 36 rats were used in the study and were randomly divided into 6 groups, of 6 rats each.

Group I: Solvent control (10 ml/kg)

Group II: Standard group; metformin $(250 \mathrm{mg} / \mathrm{kg})$

Group III: Methanol extracts of B. lacera DC; MEBL (200 mg/kg)

Group IV: Methanol extracts of B. lacera DC; MEBL (400 mg/kg)

Group V: Aqueous extracts of B. lacera DC; AEBL $(200 \mathrm{mg} / \mathrm{kg}$ )

Group VI: Aqueous extracts of B. lacera DC; AEBL ( $400 \mathrm{mg} / \mathrm{kg}$ )

The solvent control groups were treated orally with distilled water and 2 drops of Tween 80. Except that, all other groups were treated with metformin and extracts (lower and higher dose) dissolved in distilled water accordingly with the help of an oral gavage tube.

Glucose tolerance test of extracts of $B$. lacera DC aerial parts on blood glucose level of normal and streptozotocin (STZ) induced hyperglycemic rats

Oral glucose tolerance test was conducted in $12 \mathrm{~h}$ fasted rats with free access only to water. They were divided into 6 groups of six rats each as mentioned above. After half an hour administration of drugs (test and control treated) to the above-mentioned groups respectively, glucose (2 $\mathrm{g} / \mathrm{kg}$ body weight) was administered to them. The blood glucose levels were estimated before and at $0.5,1,2$ and $3 \mathrm{~h}$ of the time interval in normal and streptozotocin (STZ) induced hyperglycemic rats by using a glucometer with a guiding principle of the photometric endpoint.

Acute (single dose) effects of extracts of $B$. lacera DC aerial parts on blood glucose level of streptozotocin (STZ) treated hyperglycemic rats

According to the experimental design, drugs were administered to the $12 \mathrm{~h}$ fasted wistar albino rats orally. Blood glucose levels were estimated at $0,1,2,4,6,8,10 \mathrm{~h}$ of administration of test treated and control groups, from the tail vein, using a glucometer (Accu-check Active from Roche India Pvt. Ltd., Mumbai) [14].
Sub-acute (multi-dose) effects of extracts B. lacera DC aerial parts on blood glucose level of streptozotocin (STZ) treated hyperglycemic rats

Test drugs were administered daily to the experimental animals for a period of $30 \mathrm{~d}$. Blood glucose levels and body weights were measured at $0,5,10,15,20,25$ and $30 \mathrm{~d}$ intervals. On $30^{\text {th }} \mathrm{d}$ all the experimental rats were sacrificed by cervical decapitation and blood samples were collected by heart puncture method, then it was taken for the evaluation of serum biochemical parameters and lipid profiles. The organs such as liver and pancreas were isolated and stored in a sterile container with diluted formalin solution and kept for histopathology investigation $[11,12]$.

\section{Statistical analysis}

Statistical analysis was carried out using statistical package for the social sciences (SPSS) version 20.0 (SPSS, Inc., Chicago, USA) licensed to the institute. Summary statistics (Mean, Standard error of mean) were used to represent the data and comparison of means in between the groups were done using analysis of variance (ANOVA) followed by post-hoc analysis (Tukey's test). The results were considered statistically significant at $p$-value less than 0.05 .

\section{RESULTS AND DISCUSSION}

\section{Phytochemical screening}

The qualitative phytochemical screening of extracts of $B$. lacera revealed the presence of carbohydrates, saponins, tannins, phenols, polyphenols, flavonoids, reducing sugar, steroids, etc. alkaloids, glycosides and tannins were not detected.

\section{Acute toxicity study}

There were no signs of acute toxicity and mortality found at the highest dose of $4000 \mathrm{mg} / \mathrm{kg}$ body weight after $72 \mathrm{~h}$ of observation. Thus, we fixed our cut-off dose at $1 / 10^{\text {th }}$ of the lethal dose i.e. 400 $\mathrm{mg} / \mathrm{kg}$ body weight. Body weight were found to be normal in test groups. We selected two dose levels i.e. 200 and $400 \mathrm{mg} / \mathrm{kg}$ body weight (lower and higher dose levels) in the experiment.

\section{Effects of extracts of B. lacera DC. on hypoglycemia using normal rats}

The mean value of blood glucose level in case of methanol extract (MEBL) and aqueous extract (AEBL) of B. lacera at $400 \mathrm{mg} / \mathrm{kg}$ body weight, showed $66.00 \pm 3.51$ and $65.67 \pm 1.69 \mathrm{mg} / \mathrm{dl}$ respectively at the end of $4 \mathrm{~h}$. Marked hypoglycaemic effect was not seen even when we increased the strength of the extracts to $400 \mathrm{mg} / \mathrm{dl}$. The standard blood glucose level in case of normal rats are $50-135 \mathrm{mg} / \mathrm{dl}$ [16]. Thus, our extracts did not produce any hypoglycaemic effect which is an advantage over current medicine system (table 1).

\section{Effects of extracts of B. lacera DC. on glucose tolerance test} using normal and diabetic rats

Methanol extract of B. lacera (MEBL) $400 \mathrm{mg} / \mathrm{kg}$ produced a maximal fall of blood glucose i.e. $34.68 \pm 1.58$ at $3 \mathrm{~h}$ in normal rats as compared to other forms and dose of extracts. (table 2) Whereas in case of diabetic rats also, it produced a significant fall $(p<0.01)$ of blood glucose $(51.88 \pm 1.66)$ at $3 \mathrm{~h}$ resembling with the effects produced by standard drug metformin $(55.67 \pm 1.56)$ (table 3$)$. The aqueous extract treated groups (AEBL at 200 and $400 \mathrm{mg} / \mathrm{kg}$ body weight) exhibited less fall of blood glucose in normal rats as well as diabetic treated rats compared to the other groups. In both the cases, methanol extract $400 \mathrm{mg} / \mathrm{kg}$ showed potent blood glucose lowering effects resembling with the standard metformin treated group (table 2,3).

Effects of extracts of B. lacera DC. on acute anti-diabetic study using diabetic rats

Single dose administration of the methanol extract treated groups (MEBL) $400 \mathrm{mg} / \mathrm{kg}$ body weight to the streptozotocin (STZ) induced diabetic rats showed percentage decrease of blood glucose at the end of $8 \mathrm{~h}$ i.e. $68.04 \pm 1.19$ and metformin treated groups $73.64 \pm 0.37$. Blood glucose level of MEBL $400 \mathrm{mg} / \mathrm{kg}$ treated groups starts decreasing in blood glucose significantly $(\mathrm{p}<0.01)$ from $2 \mathrm{~h}$ onwards 
and came down to normal level $(90.33 \mathrm{mg} / \mathrm{dl} \pm 2.60)$ like standard metformin treated group. Decrease in blood glucose levels in case of AECR (200 and $400 \mathrm{mg} / \mathrm{kg}$ body weight) treated groups starts from $1 \mathrm{~h}(\mathrm{p}<0.01)$ and were very minimal and levels were not found to come down to normal levels even till the end of $8 \mathrm{~h}$ as compared to other groups (table 4).

Effects of extracts of $B$. lacera DC. on sub-acute antidiabetic study using diabetic rats

The results of the sub-acute study (multi-dose administration) summarize that metformin being the standard drug showed maximum blood glucose lowering effect i.e. $67.14 \pm 0.69$ on the $30^{\text {th }} \mathrm{d}$ of experimental period and methanol extract treated groups (MEBL) at $400 \mathrm{mg} / \mathrm{kg}$ showed resemblance in percent decrease in blood glucose i.e. $63.78 \pm 0.59$.

The blood glucose reducing power of metformin and methanol extract treated groups (MEBL) $400 \mathrm{mg} / \mathrm{kg}$ starts on $5^{\text {th }} \mathrm{d}$ significantly $(\mathrm{p}<0.01)$. While MEBL at $200 \mathrm{mg} / \mathrm{kg}$ showed significant but not remarkable blood glucose lowering $(30.40 \pm 1.79)$ as that of the higher dose. AEBL at 200 and $400 \mathrm{mg} / \mathrm{kg}$ b. w showed blood glucose lowering effect significantly i.e. $25.96 \pm 0.41$ and $32.99 \pm 1.54$ at the end of $30^{\text {th }} \mathrm{d}$ respectively which was very lower (table 5 ).
Effects of extracts of B. lacera DC. on serum lipid profile and biochemical parameters

After the drug administration of $30 \mathrm{~d}$ daily to hyperglycemic rats, the blood was collected at the end of $30^{\text {th }}$ day in ethylene diamine tetraacetic acid (EDTA) coated vials and stored for investigations of various lipid profiles, biochemical parameters etc. It was seen that low density lipoproteins (LDL), total cholesterol, very low-density lipoproteins (VLDL), triglyceride levels were decreased significantly in metformin and extracts treated groups. Whereas high density lipoproteins (HDL) level was increased significantly in metformin and extracts treated groups especially in methanol treated (MEBL) at $400 \mathrm{mg} / \mathrm{kg}$ body weight (table 6 ).

Difference in mean levels of all serum biochemical parameters of liver enzyme levels like alanine aminotransferase (ALT), aspartate aminotransferase (AST), alkaline phosphatase (ALP), bilirubin levels (like total and direct bilirubin) and serum albumin and globulin levels were found to be significantly different among the study groups (table 7). Glycated haemoglobins ( $\mathrm{HbA} 1_{\mathrm{c}}$ ) levels also showed similar difference where the difference was due to the difference in means of solvent group to metformin and methanol extract treated (MEBL) $400 \mathrm{mg} / \mathrm{kg}$ group (table 7).

Table 1: Effects of extracts of B. lacera DC, on hypoglycemia, using normal rats

\begin{tabular}{|c|c|c|c|c|c|c|}
\hline \multirow[t]{2}{*}{ Drug treatments (Dose) } & \multicolumn{5}{|c|}{ Blood glucose levels (mg/dl) } & \multirow{2}{*}{$\begin{array}{l}\text { \% decrease blood } \\
\text { glucose at the end of } 4 \mathrm{~h}\end{array}$} \\
\hline & $\mathbf{0} \mathbf{h}^{\mathbf{b}}$ & $1 \mathrm{~h}$ & $2 \mathrm{~h}$ & $3 \mathbf{h}$ & $4 \mathrm{~h}$ & \\
\hline \multirow[t]{2}{*}{ Solvent control $(10 \mathrm{ml} / \mathrm{kg})$} & 95.33 & 95.67 & 94.67 & 95.17 & 97.00 & - \\
\hline & \pm 3.32 & \pm 3.52 & \pm 2.46 & \pm 2.54 & \pm 2.35 & \\
\hline \multirow[t]{2}{*}{ Metformin (250 mg/kg) } & 98.17 & 89.83 & 77.50 & 65.00 & 55.83 & 42.79 \\
\hline & \pm 2.95 & $\pm 2.66^{\mathrm{d}}$ & $\pm 2.87 \mathrm{c}$ & $\pm 1.37^{\mathrm{c}}$ & $\pm 1.40^{\mathrm{c}}$ & \pm 2.51 \\
\hline \multirow[t]{2}{*}{ MEBL (200 mg/kg) } & 92.00 & 89.00 & 85.50 & 82.83 & 81.17 & 11.77 \\
\hline & \pm 1.88 & $\pm 2.05^{\mathrm{c}}$ & $\pm 2.49 \mathrm{c}$ & $\pm 2.39 \mathrm{c}$ & $\pm 1.82^{\mathrm{c}}$ & \pm 0.86 \\
\hline \multirow[t]{2}{*}{ MEBL (400 mg/kg) } & 88.17 & 78.17 & 73.17 & 67.50 & 66.00 & 24.33 \\
\hline & \pm 3.60 & \pm 2.18 & $\pm 2.40^{\mathrm{d}}$ & $\pm 3.84^{\mathrm{d}}$ & $\pm 3.51^{\mathrm{d}}$ & \pm 5.54 \\
\hline \multirow[t]{2}{*}{ AEBL (200 mg/kg) } & 91.50 & 89.17 & 87.33 & 86.00 & 85.00 & 7.10 \\
\hline & \pm 1.43 & $\pm 1.40^{\mathrm{c}}$ & $\pm 1.20^{\mathrm{c}}$ & $\pm 1.46^{\mathrm{c}}$ & $\pm 1.59^{c}$ & \pm 0.97 \\
\hline \multirow[t]{2}{*}{ AEBL (400 mg/kg) } & 83.83 & 77.00 & 71.00 & 66.67 & 65.67 & 20.98 \\
\hline & \pm 3.13 & \pm 1.61 & $\pm 2.39 \mathrm{~d}$ & $\pm 1.63^{\mathrm{d}}$ & $\pm 1.69^{\mathrm{d}}$ & \pm 4.03 \\
\hline F Statistics for decrease in blood sugar & - & 3.011 & 4.864 & 12.88 & 19.88 & 25.61 \\
\hline $\mathrm{p}$ value & - & 0.026 & 0.002 & $0.000 *$ & $0.000^{*}$ & $0.000^{*}$ \\
\hline
\end{tabular}

Values represent mean \pm SEM (Standard error of mean); $\mathrm{n}=6$ in each group; *Significant difference at $\alpha=0.01$ levels; ${ }^{\text {BBaseline values for }}$

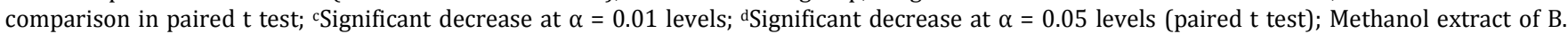
lacera (MEBL); Aqueous extract of B. lacera (AEBL).

Table 2: Effects of extracts of $B$. lacera $\mathrm{DC}$, on glucose tolerance test, using normal rats

\begin{tabular}{|c|c|c|c|c|c|c|}
\hline \multirow[t]{2}{*}{ Drug treatments (Dose) } & \multicolumn{5}{|c|}{ Blood glucose levels (mg/dl) } & \multirow{2}{*}{$\begin{array}{l}\text { \% decrease blood glucose } \\
\text { at the end of } 3 \mathrm{~h}\end{array}$} \\
\hline & $0 \mathrm{~h}$ & $0.5 \mathrm{~h}^{\mathrm{b}}$ & $1 \mathrm{~h}$ & $2 \mathrm{~h}$ & $3 \mathrm{~h}$ & \\
\hline Solvent control & 97.00 & 132.33 & 128.67 & 124.17 & 122.33 & - \\
\hline$(10 \mathrm{ml} / \mathrm{kg})$ & \pm 3.76 & \pm 1.89 & $\pm 1.02^{\mathrm{c}}$ & $\pm 1.35^{\mathrm{c}}$ & $\pm 1.59^{\mathrm{c}}$ & \\
\hline Metformin & 97.33 & 135.17 & 122.50 & 104.33 & 79.83 & 40.86 \\
\hline$(250 \mathrm{mg} / \mathrm{kg})$ & \pm 3.51 & \pm 2.12 & $\pm 1.57 \mathrm{c}$ & \pm 1.76 & $\pm 3.38^{\mathrm{d}}$ & \pm 2.72 \\
\hline MEBL & 92.67 & 126.33 & 122.00 & 117.83 & 100.33 & 20.59 \\
\hline$(200 \mathrm{mg} / \mathrm{kg})$ & \pm 3.43 & \pm 2.20 & $\pm 1.84^{\mathrm{c}}$ & $\pm 1.72^{\mathrm{c}}$ & $\pm 2.64^{\mathrm{d}}$ & \pm 1.45 \\
\hline MEBL & 86.50 & 136.00 & 124.17 & 110.17 & 88.67 & 34.68 \\
\hline$(400 \mathrm{mg} / \mathrm{kg})$ & \pm 1.67 & \pm 2.54 & $\pm 1.97 \mathrm{c}$ & $\pm 2.86^{\mathrm{c}}$ & \pm 1.02 & \pm 1.58 \\
\hline AEBL & 97.00 & 134.83 & 128.83 & 121.33 & 112.83 & 16.26 \\
\hline$(200 \mathrm{mg} / \mathrm{kg})$ & \pm 2.57 & \pm 1.60 & $\pm 1.25^{\mathrm{c}}$ & $\pm 1.20^{\mathrm{c}}$ & $\pm 1.58^{\mathrm{d}}$ & \pm 1.48 \\
\hline AEBL & 89.67 & 132.83 & 127.17 & 121.00 & 109.67 & 17.44 \\
\hline$(400 \mathrm{mg} / \mathrm{kg})$ & \pm 1.84 & \pm 1.42 & $\pm 1.30^{\mathrm{c}}$ & $\pm 1.79^{\mathrm{c}}$ & $\pm 1.69^{\mathrm{c}}$ & \pm 0.89 \\
\hline F Statistics for decrease in blood sugar & - & - & 11.84 & 17.19 & 47.07 & 56.98 \\
\hline $\mathrm{p}$ value & - & - & $0.000^{*}$ & $0.000^{*}$ & $0.000^{*}$ & $0.000^{*}$ \\
\hline
\end{tabular}

Values represent mean \pm SEM (Standard error of mean), $n=6$ animals in each group; *Significant difference at $\alpha=0.01$ levels; ${ }^{b}$ Baseline values; cSignificant decrease at $\alpha=0.01$ levels; dSignificant decrease at $\alpha=0.05$ levels (paired t-test); Methanol extract of B. lacera (MEBL); Aqueous extract of B. lacera (AEBL). 
Table 3: Effects of extracts of $B$. lacera DC, on glucose tolerance test using hyperglycemic rats

\begin{tabular}{|c|c|c|c|c|c|c|}
\hline \multirow{2}{*}{$\begin{array}{l}\text { Drug treatments } \\
\text { (Dose) }\end{array}$} & \multicolumn{5}{|c|}{ Blood glucose levels (mg/dl) } & \multirow{2}{*}{$\begin{array}{l}\% \text { decrease in blood glucose } \\
\text { at the end of } 3 \mathrm{~h}\end{array}$} \\
\hline & $\mathbf{O} \mathbf{h}$ & $0.5 \mathrm{~h}^{\mathrm{b}}$ & $1 \mathrm{~h}$ & $2 \mathrm{~h}$ & $3 \mathbf{h}$ & \\
\hline Solvent control & 279.50 & 303.33 & 307.00 & 308.00 & 309.50 & - \\
\hline$(10 \mathrm{ml} / \mathrm{kg})$ & \pm 5.18 & \pm 4.47 & \pm 6.52 & \pm 6.30 & \pm 7.11 & \\
\hline Metformin & 275.00 & 301.17 & 221.83 & 179.17 & 132.83 & 55.67 \\
\hline$(250 \mathrm{ml} / \mathrm{kg})$ & \pm 14.64 & \pm 14.11 & $\pm 16.90^{c}$ & $\pm 10.92^{\mathrm{c}}$ & $\pm 4.785^{c}$ & \pm 1.56 \\
\hline MEBL & 286.33 & 314.67 & 291.67 & 281.50 & 241.17 & 23.31 \\
\hline (200 mg/kg) & \pm 6.40 & \pm 6.84 & \pm 6.18 & \pm 6.22 & $\pm 5.89^{\mathrm{c}}$ & \pm 1.36 \\
\hline MEBL & 327.33 & 350.17 & 268.33 & 206.33 & 169.33 & 51.88 \\
\hline$(400 \mathrm{mg} / \mathrm{kg})$ & \pm 38.27 & \pm 38.63 & \pm 29.49 & $\pm 19.34^{\mathrm{c}}$ & $\pm 20.67 \mathrm{c}$ & \pm 1.66 \\
\hline AEBL & 282.33 & 310.33 & 281.67 & 265.33 & 248.67 & 19.93 \\
\hline (200 mg/kg) & \pm 5.97 & \pm 6.95 & \pm 8.68 & $\pm 8.84^{\mathrm{c}}$ & $\pm 7.37^{\mathrm{c}}$ & \pm 0.95 \\
\hline AEBL & 304.00 & 339.83 & 292.17 & 269.50 & 238.67 & 29.93 \\
\hline (400 mg/kg) & \pm 21.64 & \pm 19.42 & \pm 19.98 & $\pm 18.17^{\mathrm{c}}$ & $\pm 17.26^{\mathrm{c}}$ & \pm 1.99 \\
\hline F Statistics for decrease in blood sugar & - & - & 32.34 & 24.53 & 46.88 & 194.11 \\
\hline $\mathrm{p}$ value & - & - & $0.000 *$ & $0.000^{*}$ & $0.000^{*}$ & $0.000^{*}$ \\
\hline
\end{tabular}

Values represent mean \pm SEM (Standard error of mean), $n=6$ animals in each group, ${ }^{* *}$ Significant difference at $\alpha=0.01$ levels; ${ }^{b}$ Baseline values for comparison in paired t-test, cSignificant decrease at $\alpha=0.01$ levels; ${ }^{d}$ Significant decrease at $\alpha=0.05$ levels (paired t-test); Methanol extract of B. lacera (MEBL); Aqueous extract of B. lacera (AEBL).

Table 4: Effects of extracts of $B$. lacera $\mathrm{DC}$, on acute anti-diabetic study using diabetic rats

\begin{tabular}{|c|c|c|c|c|c|c|c|c|}
\hline \multirow[t]{2}{*}{ Drug treatments (Dose) } & \multicolumn{7}{|c|}{ Blood glucose levels (mg/dl) } & \multirow{2}{*}{$\begin{array}{l}\text { \% decrease blood } \\
\text { glucose at the end of } 8 \mathrm{~h}\end{array}$} \\
\hline & $\mathbf{O} \mathbf{h}^{\mathbf{b}}$ & $1 \mathrm{~h}$ & $2 \mathrm{~h}$ & $3 \mathbf{h}$ & $4 \mathrm{~h}$ & $6 \mathrm{~h}$ & $8 \mathrm{~h}$ & \\
\hline Solvent control & 265.83 & 271.83 & 277.83 & 284.83 & 290.17 & 298.67 & 309.00 & - \\
\hline$(10 \mathrm{ml} / \mathrm{kg})$ & \pm 10.11 & \pm 9.83 & $\pm 9.66^{c}$ & $\pm 9.60^{c}$ & $\pm 8.51^{\mathrm{c}}$ & $\pm 7.99^{\mathrm{c}}$ & $\pm 9.21^{\mathrm{c}}$ & \\
\hline Metformin & 306.33 & 261.33 & 229.00 & 169.00 & 125.17 & 105.33 & 80.50 & 73.64 \\
\hline$(250 \mathrm{mg} / \mathrm{kg})$ & \pm 14.80 & \pm 17.86 & $\pm 17.54^{\mathrm{c}}$ & $\pm 12.36^{\mathrm{c}}$ & $\pm 8.79 \mathrm{c}$ & $\pm 6.69^{c}$ & $\pm 2.85^{\mathrm{c}}$ & \pm 0.37 \\
\hline MEBL & 287.33 & 270.00 & 250.83 & 226.67 & 203.83 & 185.33 & 168.00 & 41.49 \\
\hline$(200 \mathrm{mg} / \mathrm{kg})$ & \pm 6.53 & $\pm 5.82^{\mathrm{c}}$ & $\pm 5.15^{c}$ & $\pm 3.76^{c}$ & $\pm 4.49^{\mathrm{c}}$ & $\pm 4.54^{\mathrm{c}}$ & $\pm 5.04^{\mathrm{c}}$ & \pm 1.53 \\
\hline MEBL & 285.00 & 260.83 & 229.00 & 195.67 & 156.33 & 113.17 & 90.33 & 68.04 \\
\hline$(400 \mathrm{mg} / \mathrm{kg})$ & \pm 15.57 & \pm 21.08 & $\pm 19.35^{c}$ & $\pm 19.09 \mathrm{c}$ & $\pm 13.85^{c}$ & $\pm 5.21^{\mathrm{c}}$ & $\pm 2.60^{c}$ & \pm 1.19 \\
\hline AEBL & 289.67 & 276.33 & 257.83 & 218.50 & 200.67 & 192.50 & 219.17 & 24.38 \\
\hline$(200 \mathrm{mg} / \mathrm{kg})$ & \pm 7.14 & $\pm 7.09^{\mathrm{c}}$ & $\pm 8.12^{\mathrm{c}}$ & $\pm 6.68^{\mathrm{c}}$ & $\pm 6.99^{c}$ & $\pm 10.71^{\mathrm{c}}$ & $\pm 6.89^{c}$ & \pm 0.94 \\
\hline AEBL & 319.17 & 289.00 & 266.83 & 232.50 & 216.17 & 198.67 & 185.83 & 42.45 \\
\hline$(400 \mathrm{mg} / \mathrm{kg})$ & \pm 20.69 & $\pm 21.97 \mathrm{c}$ & $\pm 22.57 \mathrm{c}$ & $\pm 26.67 \mathrm{c}$ & $\pm 27.44 \mathrm{c}$ & $\pm 24.85^{\mathrm{c}}$ & $\pm 24.51^{\mathrm{c}}$ & \pm 5.09 \\
\hline $\begin{array}{l}\text { F Statistics for decrease in blood } \\
\text { sugar }\end{array}$ & - & 9.04 & 26.48 & 34.69 & 50.69 & 68.47 & 82.50 & 187.85 \\
\hline$p$ value & - & $0.000^{*}$ & $0.000^{*}$ & $0.000^{*}$ & $0.000^{*}$ & $0.000 *$ & $0.000^{*}$ & $0.000^{*}$ \\
\hline
\end{tabular}

Values represent mean \pm SEM (Standard error of mean), $n=6$ animals in each group; **Significant difference at $\alpha=0.01$ levels; bBaseline values for comparison in paired t-test; cSignificant decrease at $\alpha=0.01$ levels; ${ }^{\text {d}}$ Significant decrease at $\alpha=0.05$ levels (paired t-test); Methanol extract of B. lacera (MEBL); Aqueous extract of B. lacera (AEBL).

Table 5: Effects of extracts of $B$. lacera DC, on sub-acute antidiabetic study using diabetic rats

\begin{tabular}{|c|c|c|c|c|c|c|c|c|}
\hline \multirow[t]{2}{*}{ Drug treatments (Dose) } & \multicolumn{7}{|c|}{ Blood glucose levels (mg/dl) } & \multirow{2}{*}{$\begin{array}{l}\text { \% decrease blood glucose } \\
\text { at the end of } 30^{\text {th }} \mathrm{d}\end{array}$} \\
\hline & 0-d $\mathbf{d}^{\mathbf{b}}$ & $5^{\text {th }} \mathbf{d}$ & $10^{\text {th }} \mathrm{d}$ & $15^{\text {th }} d$ & $20^{\text {th }} d$ & $25^{\text {th }} d$ & $30^{\text {th }} \mathrm{d}$ & \\
\hline Solvent control & 282.17 & 293.00 & 303.00 & 315.67 & 327.00 & 335.67 & 347.17 & - \\
\hline$(10 \mathrm{ml} / \mathrm{kg})$ & \pm 6.79 & \pm 8.38 & $\pm 8.63^{c}$ & $\pm 9.62^{c}$ & $\pm 9.69^{c}$ & $\pm 10.10^{c}$ & $\pm 7.28^{\mathrm{c}}$ & \\
\hline Metformin & 280.83 & 252.67 & 215.83 & 177.17 & 156.00 & 135.00 & 92.17 & 67.14 \\
\hline$(250 \mathrm{mg} / \mathrm{kg})$ & \pm 4.90 & $\pm 8.77 \mathrm{c}$ & $\pm 9.40^{\mathrm{c}}$ & $\pm 10.76^{c}$ & $\pm 12.85^{\mathrm{c}}$ & $\pm 10.93^{c}$ & $\pm 1.54^{\mathrm{c}}$ & \pm 0.69 \\
\hline MEBL & $289.83 \pm 9.83$ & 281.67 & 272.83 & 261.83 & 249.00 & 229.17 & 201.83 & 30.40 \\
\hline$(200 \mathrm{mg} / \mathrm{kg})$ & & \pm 9.62 & $\pm 11.30^{c}$ & $\pm 12.74 \mathrm{c}$ & $\pm 13.32^{\mathrm{c}}$ & $\pm 12.58^{c}$ & $\pm 8.87 \mathrm{c}$ & \pm 1.79 \\
\hline MEBL & $289.83 \pm 2.71$ & 261.67 & 234.17 & 205.83 & 173.50 & 134.17 & 105.00 & 63.78 \\
\hline$(400 \mathrm{mg} / \mathrm{kg})$ & & $\pm 5.11^{\mathrm{c}}$ & $\pm 5.82^{\mathrm{c}}$ & $\pm 5.68^{c}$ & $\pm 3.50^{c}$ & $\pm 5.19^{c}$ & $\pm 2.05^{\mathrm{c}}$ & \pm 0.59 \\
\hline AEBL & $305.33 \pm 3.28$ & 300.17 & 292.33 & 286.00 & 264.83 & 244.00 & 226.00 & 25.96 \\
\hline$(200 \mathrm{mg} / \mathrm{kg})$ & & $\pm 3.34^{\mathrm{c}}$ & $\pm 2.20^{\mathrm{c}}$ & $\pm 2.07^{\mathrm{c}}$ & $\pm 3.03^{\mathrm{c}}$ & $\pm 4.79^{\mathrm{c}}$ & $\pm 1.65^{\mathrm{c}}$ & \pm 0.41 \\
\hline AEBL & $283.33 \pm 3.09$ & 274.33 & 265.33 & 250.50 & 231.33 & 217.00 & 189.83 & 32.99 \\
\hline$(400 \mathrm{mg} / \mathrm{kg})$ & & $\pm 3.51^{\mathrm{c}}$ & \pm 5.32 & $\pm 8.50^{\mathrm{c}}$ & $\pm 7.27^{\mathrm{c}}$ & $\pm 6.82^{\mathrm{c}}$ & $\pm 4.56^{c}$ & \pm 1.54 \\
\hline $\begin{array}{l}\text { F Statistics for decrease in blood } \\
\text { sugar }\end{array}$ & - & 19.59 & 34.81 & 49.53 & 57.40 & 91.52 & 344.29 & 369.08 \\
\hline $\mathrm{p}$ value & - & $0.000^{*}$ & $0.000^{*}$ & $0.000^{*}$ & $0.000^{*}$ & $0.000^{*}$ & $0.000^{*}$ & $0.000^{*}$ \\
\hline
\end{tabular}

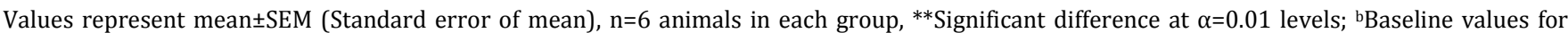
comparison in paired t-test, cSignificant decrease at $\alpha=0.01$ levels; dSignificant decrease at $\alpha=0.05$ levels (paired t-test); Methanol extract of B. lacera (MEBL); Aqueous extract of B. lacera (AEBL).

\section{Histopathology evaluation}

The liver and pancreas of all the treated groups of rats were isolated on the $30^{\text {th }} \mathrm{d}$ of sub-acute antidiabetic study and stored in a sterile container with $20 \%$ formaldehyde solution for histopathology as an essential part of pre-clinical safety aspects. The sections were stained in hematoxylin and eosin and Photos of liver and pancreas were taken at 40X and 100X magnification respectively. Methanol extract treated groups (MEBL) $400 \mathrm{mg} / \mathrm{kg}$ showed potent beneficial effects against Diabetes mellitus (DM) in case of liver which shows 
normal hepatocytes architecture and there was no necrosis or malignancy found among all. Also in pancreas sections, the results were similar as above i.e. methanol extract treated groups (MEBL) at $400 \mathrm{mg} / \mathrm{kg}$ showed regular shaped islet with maximum number of beta cells (fig. $1(\mathrm{~A}-\mathrm{H})$ ). The yellow arrow in liver section indicating the hepatocytes and the green arrow indicating the central vein. The blue arrow in the pancreas indicating the Islet of Langerhans and the black arrows indicating the beta cells (fig. $1(\mathrm{~A}-\mathrm{H})$ ).

Table 6: Effects of extracts of $B$. lacera DC, on serum lipid profile investigation

\begin{tabular}{|c|c|c|c|c|c|c|}
\hline \multirow{2}{*}{$\begin{array}{l}\text { Drug treatments } \\
\text { (Dose) }\end{array}$} & \multicolumn{6}{|c|}{ Serum lipid profile (mg/dl) } \\
\hline & LDL & HDL & Triglycerides & VLDL & Total cholesterol & Phospholipids \\
\hline Solvent control & 108.08 & 28.12 & 175.12 & 35.02 & 171.21 & 207.55 \\
\hline$(10 \mathrm{ml} / \mathrm{kg})$ & \pm 3.56 & \pm 2.89 & \pm 5.39 & \pm 1.08 & \pm 3.45 & \pm 3.49 \\
\hline Metformin & 30.13 & 49.92 & 69.98 & 13.99 & 94.05 & 110.67 \\
\hline (250 mg/kg) & \pm 1.87 & \pm 2.01 & \pm 2.46 & \pm 0.49 & \pm 3.49 & \pm 2.90 \\
\hline MEBL & 90.36 & 28.16 & 128.71 & 25.74 & 144.27 & 183.05 \\
\hline$(200 \mathrm{mg} / \mathrm{kg})$ & \pm 4.18 & \pm 1.26 & \pm 2.82 & \pm 0.56 & \pm 4.25 & \pm 2.40 \\
\hline MEBL & 29.73 & 53.30 & 72.00 & 14.40 & 97.43 & 126.77 \\
\hline (400 mg/kg) & \pm 2.46 & \pm 2.99 & \pm 1.30 & \pm 0.26 & \pm 3.54 & \pm 3.55 \\
\hline AEBL & 109.38 & 20.91 & 104.20 & 20.84 & 151.13 & 163.02 \\
\hline$(200 \mathrm{mg} / \mathrm{kg})$ & \pm 3.05 & \pm 0.74 & \pm 4.67 & \pm 0.93 & \pm 3.90 & \pm 3.75 \\
\hline AEBL & 64.48 & 35.75 & 106.13 & 21.23 & 121.46 & 117.63 \\
\hline$(400 \mathrm{mg} / \mathrm{kg})$ & \pm 2.61 & \pm 2.09 & \pm 1.38 & \pm 0.28 & \pm 2.81 & \pm 2.07 \\
\hline F Statistics & 142.67 & 36.34 & 134.50 & 134.51 & 73.59 & 160.97 \\
\hline $\mathrm{p}$ value & $0.000^{* *}$ & $0.000 * *$ & $0.000 * *$ & $0.000 * *$ & $0.000 * *$ & $0.000 * *$ \\
\hline
\end{tabular}

Values represent mean \pm SEM(Standard error of the mean), n=6 in each group. LDL, Lower density lipids; HDL, Higher density lipids; VLDL, Very lower density lipids; ** Significant difference at $\alpha=0.01$ levels; Methanol extract of B. lacera (MEBL); Aqueous extract of B. lacera (AEBL).

Table 7: Effects of extracts of $B$. lacera DC, on serum biochemical parameters and $\mathrm{HbA1}$ clevels

\begin{tabular}{|c|c|c|c|c|c|c|c|c|c|}
\hline \multirow{2}{*}{$\begin{array}{l}\text { Drug treatments } \\
\text { (Dose) }\end{array}$} & \multicolumn{9}{|c|}{ Serum biochemical parameters } \\
\hline & $\begin{array}{l}\text { AST }^{\#} \\
(\mathbf{u} / \mathbf{l})\end{array}$ & $\begin{array}{l}\text { ALT }^{\#} \\
(\mathbf{u} / \mathbf{l})\end{array}$ & $\begin{array}{l}\text { ALP } \\
(u / l)\end{array}$ & $\begin{array}{l}\text { BT } \\
(\mathrm{mg} / \mathrm{d})\end{array}$ & $\begin{array}{l}\text { BD } \\
\text { (mg/dl) }\end{array}$ & $\begin{array}{l}\text { TP } \\
\text { (g/dl) }\end{array}$ & $\begin{array}{l}\text { Albumin } \\
\text { (g/dl) }\end{array}$ & $\begin{array}{l}\text { Globulin } \\
\text { (g/dl) }\end{array}$ & $\begin{array}{l}\text { HbA1 }{ }^{\wedge} \\
(\%)\end{array}$ \\
\hline Solvent control & 96.71 & 56.89 & $207.33 \pm 13.37$ & 1.44 & 0.21 & 3.78 & 3.20 & 0.58 & 7.37 \\
\hline$(10 \mathrm{ml} / \mathrm{kg})$ & \pm 17.61 & \pm 4.09 & & \pm 0.05 & \pm 0.02 & \pm 0.22 & \pm 0.12 & \pm 0.22 & \pm 0.10 \\
\hline Metformin & 27.34 & 22.69 & $132.91 \pm 2.27$ & 0.74 & 0.35 & 5.46 & 4.27 & 1.19 & 4.28 \\
\hline$(250 \mathrm{mg} / \mathrm{kg})$ & \pm 1.18 & \pm 0.84 & & \pm 0.06 & \pm 0.05 & \pm 0.08 & \pm 0.12 & \pm 0.17 & \pm 0.09 \\
\hline MEBL & 34.97 & 49.36 & $162.98 \pm 2.63$ & 1.38 & 0.85 & 3.61 & 2.89 & 0.71 & 6.35 \\
\hline (200 mg/kg) & \pm 1.97 & \pm 1.16 & & \pm 0.02 & \pm 0.05 & \pm 0.11 & \pm 0.05 & \pm 0.13 & \pm 0.10 \\
\hline MEBL & 25.79 & 23.43 & $136.93 \pm 4.03$ & 0.61 & 0.38 & 4.82 & 3.61 & 1.21 & 4.82 \\
\hline$(400 \mathrm{mg} / \mathrm{kg})$ & \pm 0.99 & \pm 1.45 & & \pm 0.03 & \pm 0.05 & \pm 0.25 & \pm 0.18 & \pm 0.19 & \pm 0.10 \\
\hline AEBL & 56.83 & 63.73 & $169.89 \pm 4.69$ & 1.37 & 1.07 & 3.57 & 2.71 & 0.86 & 6.89 \\
\hline$(200 \mathrm{mg} / \mathrm{kg})$ & \pm 4.53 & \pm 3.17 & & \pm 0.04 & \pm 0.07 & \pm 0.11 & \pm 0.09 & \pm 0.16 & \pm 0.11 \\
\hline AEBL & 41.82 & 39.30 & $155.26 \pm 2.49$ & 1.02 & 0.72 & 4.19 & 2.88 & 1.31 & 6.32 \\
\hline$(400 \mathrm{mg} / \mathrm{kg})$ & \pm 2.04 & \pm 2.21 & & \pm 0.17 & \pm 0.12 & \pm 0.13 & \pm 0.24 & \pm 0.16 & \pm 0.16 \\
\hline F Statistics & 12.58 & 49.37 & 18.51 & 20.46 & 25.40 & 21.91 & 15.67 & 2.94 & 113.28 \\
\hline p value & $0.000^{* *}$ & $0.000^{* *}$ & $0.000 * *$ & $0.000 * *$ & $0.000 * *$ & $0.000^{* *}$ & $0.000^{* *}$ & $0.000 * *$ & $0.000^{* *}$ \\
\hline
\end{tabular}

Values represent mean \pm SEM(Standard error of the mean), $\mathrm{n}=6$ in each group; ALAT, Alanine aminotransferase; ASAT, Aspartate aminotransferase; ALP, Alkaline Phosphatase; BT, Bilirubin total; BD, Bilirubin direct; TP, Total protein; \# in $\mathrm{u}^{\wedge} \mathrm{l}^{\wedge}{ }^{\wedge}$ in $\%$. ${ }^{* *}$ Significant difference at $\alpha=0.01$ level; Methanol extract of B. lacera (MEBL); Aqueous extract of B. lacera (AEBL).
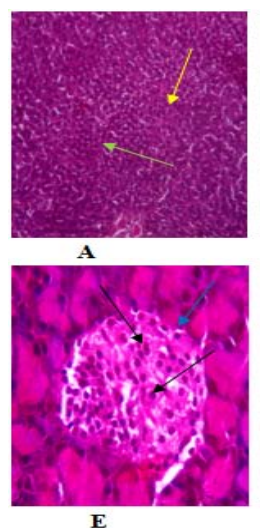

$\mathbf{E}$

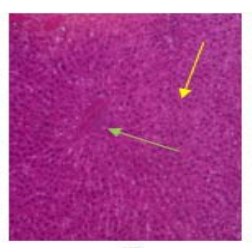

$\mathbf{B}$

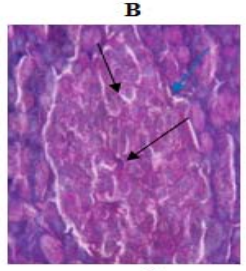

$\mathbf{F}$
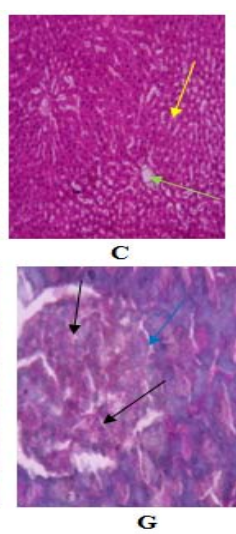
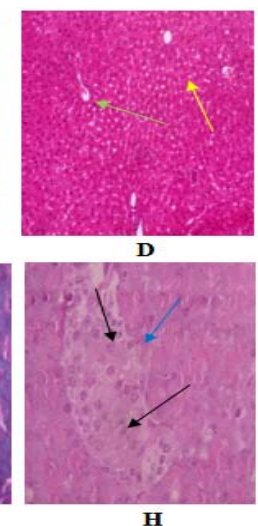

Fig. 1(A-H): Histopathological findings of liver and pancreas in diabetic rats, A: Rat Liver section of methanol extract of B. lacera (MEBL) 200 $\mathrm{mg} / \mathrm{kg}$ treated diabetic rat, B: Rat Liver section of methanol extract of B. lacera (MEBL) $400 \mathrm{mg} / \mathrm{kg}$ treated diabetic rat, C: Rat Liver section of aqueous extract of $B$. lacera (AEBL) $200 \mathrm{mg} / \mathrm{kg}$ treated with diabetic rat, D: Rat Liver section of aqueous extract of B. lacera (AEBL) $400 \mathrm{mg} / \mathrm{kg}$ treated diabetic rat, E: Rat Pancreas section of methanol extract of B. lacera (MEBL) $200 \mathrm{mg} / \mathrm{kg}$ treated diabetic rat, F: Rat Pancreas section of methanol extract of B. lacera (MEBL) $400 \mathrm{mg} / \mathrm{kg}$ treated diabetic rat, G: Rat Pancreas section of aqueous extract of B. lacera (AEBL) 200 $\mathrm{mg} / \mathrm{kg}$ treated diabetic rat, $\mathrm{H}$ : Rat Pancreas section of aqueous extract of B. lacera (AEBL) $400 \mathrm{mg} / \mathrm{kg}$ treated diabetic rat; All were stained with Eosin andGomori, Rat Liver sections are of 40X and Rat Pancreas sections are of 100X magnification power 
In the present study, we evaluated the antidiabetic effects of aerial parts of extracts of Blumea lacera DC in streptozotocin induced hyperglycemic rats. The single dose administration of extracts of $B$. lacera did not show any hypoglycemic effects in normal rats which indicates that the plant does not have any hypoglycemic side effects. In diabetic rats, the singe administration of B. lacera extracts produce a significant dose dependent blood glucose lowering effects at the end of $8 \mathrm{~h}$ especially the methanol extract treated groups (MEBL) $400 \mathrm{mg} / \mathrm{kg}$ showing the maximum as compared to other treated groups $(\mathrm{p}<0.01)$ (table 4$)$. In sub-acute antidiabetic study, the daily administration of all the extracts showed significant lowering of blood glucose $(\mathrm{p}<0.01)$ but methanol extract treated groups (MEBL) $400 \mathrm{mg} / \mathrm{kg}$ showed maximum lowering of blood glucose at the end of $30 \mathrm{~d}$ (table 5). The decreased function of alanine aminotransferase (ALT) and aspartate aminotransferase (AST) was found in case of metformin and methanol extract treated groups which means a clear indication of restoration or normalisation of liver function (table 7). Also, the glycated haemoglobin was found to be lower significantly in case of methanol extract treated and metformin treated groups compared to the solvent control group which indicates the secretion of insulin from the existing beta cells of islets of Langerhans (table 7). There was decrease in the total cholesterol, low density lipoproteins (LDL), very low-density lipoproteins (VLDL) and increase in the value of high density lipoproteins (HDL) found in methanol treated and metformin treated groups which would be beneficial in the management of diabetes (table 6). From the histopathological evaluation, it was also seen that the liver sections of methanol extract treated groups were having no inflammation and necrosis or derangements in structure as compared to the aqueous treated groups (fig. A, B, C, D). In case of pancreatic sections of aqueous treated groups, there was irregularity in the shape of islet of Langerhans and decrease in the count of beta cells due to lesser blood glucose lowering effects (fig. G and H). But the Methanol treated groups was found to be increased in the number of beta cells and with having a regular shape of islet (fig. E and F).

The mechanism through which the methanol extract possess antidiabetic potential could be due to extrapancreatic effects and secretion of insulin from the existing beta cells of the pancreas. More detailed study on the chemical constituents should be investigated for exploration of the exact mechanism of action.

\section{CONCLUSION}

The methanol extracts of aerial parts of Blumea lacera (MEBL 400 $\mathrm{mg} / \mathrm{kg}$ ) were found to have potent antidiabetic activity in streptozotocin (STZ) induced diabetic rats. Also, there was no necrosis or histopathological injury found in methanol extract treated diabetic groups. Further detailed study of the exact mechanism of action behind its antidiabetic effects can show promising direction for new therapeutic molecules for the treatment of Diabetes Mellitus (DM).

\section{ACKNOWLEDGEMENT}

The authors are grateful to Prof. Amit Banerjee, Vice-chancellor and Prof. Sudam Ch. Si, Dean of School of Pharmaceutical Sciences, Siksha ' $O$ ' Anusandhan University, Bhubaneswar for providing facilities and necessary advice in the realization of this research work.

Diptirani Rath: Performance of whole experiments

Snigdha Rani Panigrahy: Correction of manuscript

Sandeep Kumar Panigrahi: Statistical analysis and interpretation

Durga Madhab Kar: Design of protocol of the study
Laxmidhar Maharana: Supervision of experiments

\section{CONFLICT OF INTERESTS}

Authors declare that we have no conflict of interest

\section{REFERENCES}

1. M Vijayan, R Jose, S Jose, S Abraham, J Joy. Study on quality of life assessment in diabetic retinopathy among patients with type 2 diabetic patients. Asian J Pharma Clin Res 2017;10:10-3.

2. K Arunachalam, T Parimelazhagan. Antidiabetic activity of Ficus amplissima Smith bark extract in streptozotocin-induced diabetic rats. J Ethnopharmacol 2013;147:302-10.

3. R Kant, R Bodla, G Kapoor, R Bhutani. Development and validation of novel spectrophotometric methods for simultaneous estimation of pioglitazone and metformin in bulk and fixed dosage forms by area under the curve and dual wavelength mode. Int J Appl Pharm 2016;8:48-53.

4. C Khare. Indian Medicinal Plants-An illustrated dictionary New Delhi: Springer; 2007.

5. M Salahuddin. Medicinal plants used by a traditional ayurvedic practitioner at an asadnagar village in narsingdi district, Bangladesh. J Pharmacogn Phytochem 2015;4:269-75.

6. UP Singh, AK Singh, RP Sarathy. Effect of methanolic extracts of Blumea Eriantha DC. leaves on protein metabolism and marker enzymes in streptozotocin-induced hyperglycemic animals. Int J Pharm Pharm Sci 2012;4:1-4.

7. MN Hasan, MH Rahman, R Guo, A Hirashima. Hypoglycemic activity of methanolic leaf extract of Blumea lacera in swissalbino mice. Asian Pac J Trop Dis 2015;195-8.

8. M Ibrahim, A Khair, M Ibrahim, Q Ahsan, Z Homa. Pharmacological activities of Blumea lacera (Burm. f) DC: a medicinal plant of Bangladesh plant of Bangladesh. Br J Pharm Res 2014;1808:195-8.

9. S Ram, VS Sinha. Qualitative phytochemical analysis of some plants uses to cure Malaria in Kolhan region, of Jharkhand, India. J Med Plants Stud 2015;3:60-2.

10. M Kaur, R Yadav. Pharmacognostic, ethnopharmacological, phytochemical and pharmacological profile of wild Guava i.e. Careya arborea Roxb. Int J Curr Pharm Res 2017;9:2-8.

11. OECD/OCDE. Acute oral toxicity-Acute toxic class method; 2001.

12. MC Garcia-Galicia, E Burgueno Tapia, A Romero-Rojas, JC García-Zebadúa, C Ordaz-Pichardo, J Cornejo-Garrido. The antihyperglycemic effect, inhibition of inflammatory cytokines expression, and histopathology profile in streptozotocininduced diabetic rats treated with Arracacia tolucensis aerialparts extracts. J Ethnopharmacol 2014;152:91-8.

13. D Rath, DM Kar, SK Panigrahi, L Maharana. Antidiabetic effects of Cuscuta reflexa Roxb. in streptozotocin-induced diabetic rats. J Ethnopharmacol 2016;192:442-9.

14. M Aslan, N Orhan, DD Orhan, F Ergun. Hypoglycemic activity and antioxidant potential of some medicinal plants traditionally used in turkey for diabetes. J Ethnopharmacol 2010;128:384-9.

15. MC Tchamadeu. Hypoglycaemic effects of Mammea africana (Guttiferae) in diabetic rats. J Ethnopharmacol 2010;127:368-72.

16. CPCSEA. CPCSEA guidelines for laboratory animal facility. Indian J Pharmacol 2003;35:257-74.

\section{How to cite this article}

- Diptirani Rath, Snigdha Rani Panigrahy, Sandeep Kumar Panigrahi, Durga Madhab Kar, Laxmidhar Maharana. Antidiabetic effect of extracts of Blumea lacera DC. in streptozotocin induced hyperglycemic rats. Int J Pharm Pharm Sci 2017;9(10):218-223. 